\title{
VISUALIZACIÓN WEB INTERACTIVA PARA EL ANÁLISIS DEL CHATTER EN LAMINACIÓN EN FRÍO
}

\author{
Daniel Pérez López, Abel Alberto Cuadrado Vega, Ignacio Díaz Blanco \\ Área de Ingeniería de Sistemas y Automática. Universidad de Oviedo \\ Campus Universitario de Gijón, s/n. \\ \{dperez, cuadrado\}@isa.uniovi.es, idiaz@uniovi.es
}

\section{Resumen}

La laminación de productos de acero es un proceso dentro del campo de la metalurgia donde dos o más pares de rodillos reducen el espesor de una banda de acero para producir un material de espesor uniforme. A pesar de que se ha estudiado ampliamente durante muchos años, hay problemas impredecibles que pueden aparecer y afectar a la calidad del producto final. Uno de ellos es el denominado chatter, una potente vibración autoexcitada que aparece súbitamente y limita la productividad. En este artículo se considera un enfoque de analítica visual para el análisis del chatter que ayude a descubrir y entender los factores y condiciones en las cuales aparece el chatter. Se presenta una interfaz web interactiva que permite explorar una proyección de las condiciones dinámicas y visualizar detalles correspondientes a cada episodio de chatter. Se expone finalmente un caso de validación para probar la herramienta con datos reales, donde estados normales y de chatter se identificaron automáticamente.

Palabras clave: Modelos de datos, sistemas de soporte a la decisión, sistemas expertos.

\section{INTRODUCCIÓN}

El proceso de laminación transforma la forma de un material de acero por medio de una reducción del espesor haciéndolo pasar entre dos o más pares de rodillos alojados en una caja que les sirve de soporte. Este proceso es diferente dependiendo de la temperatura del material laminado. Precisamente, un tren de laminación en frío [12] produce productos acabados más finos con un grosor de salida uniforme, llevado a cabo comúnmente de manera continua a través de varias cajas en los trenes de laminación en frío de tipo tándem. A pesar de que éste es un proceso universal en la elaboración de metales, todavía existen problemas que causan pérdidas económicas en los trenes modernos de laminación en frío. Además, las condiciones bajo las cuales surgen estos problemas no se comprenden completamente, lo que dificulta su prevención.
Uno de los principales problemas que presentan es una vibración autoexcitada denominada chatter, que aparece en los procesos de laminación, provocando variaciones de espesor inadmisibles en la superficie final de la banda, tal como se explica en [16]. La disminución de la velocidad de laminación para mitigar este efecto implica una pérdida de productividad que hace que el chatter no solo sea un problema industrial, sino también un problema económico. El análisis de chatter requiere la comprensión de las condiciones que conducen a la inestabilidad del proceso. Las interacciones dinámicas entre los fenómenos estructurales en el tren y el propio proceso de deformación de la banda se han estudiado utilizando modelos teóricos $[9,1,21,10,23]$. Estos modelos pueden llegar a ser complejos de implementar, o bien basarse en ciertos supuestos que simplifican el problema real.

Las tecnologías recientes facilitan la adquisición de datos de cualquier proceso y su almacenamiento masivo en bases de datos. Un análisis apropiado puede revelar cierta intuición acerca de problemas complejos y servir de apoyo al analista en la adopción de estrategias de actuación. Los algoritmos de análisis inteligente de datos (IDA) extraen información automáticamente de los datos permitiendo descubrir conocimiento nuevo. Un enfoque particularmente interesante es la reducción de la dimensionalidad (DR). Las técnicas DR permiten encontrar estructuras latentes de baja dimensionalidad en espacios de datos de alta dimensión, definiendo un mapeo que permite proyectar puntos de alta dimensión en un espacio latente de baja dimensión -típicamente 2D o 3D- que puede visualizarse y permite la implementación de mecanismos de interacción. Se puede encontrar una revisión detallada de las técnicas DR en el libro [8].

La presentación visual de los resultados de estos algoritmos es una excelente forma de comunicación [19] que puede mejorar la comprensión del problema y sugerir una actuación más rápida. Los mecanismos de interacción introducen al humano en el proceso de análisis, permitiéndole incorporar conocimiento de dominio del problema durante la exploración de datos, en lugar de usar representa- 
ciones estáticas. Los métodos de analítica visual (VA) permiten explotar la sinergia entre los algoritmos de análisis de datos inteligente, las técnicas de visualización y la interacción para apoyar al usuario en la consecución de un análisis eficiente. El enfoque VA se basa en el razonamiento analítico a través de interfaces interactivas, como se explica en $[15]$ y $[6]$.

En este artículo se propone un análisis del chatter utilizando el enfoque VA, permitiendo al usuario explorar las diferentes condiciones dinámicas del proceso. Esto se realiza a través de una interfaz web que permite el análisis de datos reales resultantes de algoritmos que separan comportamientos dinámicos de condiciones normales y de fallo (chatter). Los comportamientos dinámicos, caracterizados por vectores de características de alta dimensión son proyectados en un espacio latente 2D usando un algoritmo DR, y representados en una interfaz web interactiva sobre la que el usuario puede explorarlos y obtener detalles bajo demanda, como el espectrograma. El artículo está organizado como sigue: en la sección 2 se revisan trabajos previos que analizan el proceso de laminación; en la sección 3 se explica el análisis de datos para tratar el fallo del chatter; en la sección 4 se describe un caso de estudio real como método de validación; finalmente, en la sección 5 se exponen las conclusiones y se sugieren las líneas para el trabajo futuro.

\section{TRABAJO RELACIONADO}

Los fenómenos de vibración aparecen en procesos de laminación como resultado de las interacciones dinámicas producidas [16]. Hay dos modos principales de vibración vertical: el modo de tercera octava (120-250 Hz) y el modo de quinta octava (500-700 Hz), siendo el primero de ellos el más dañino, que ocurre de forma repentina acumulando una gran cantidad de energía en pocos segundos. Identificamos aquí al chatter con este modo de vibración de tercera octava.

Varios trabajos han estudiado este fenómeno previamente. Los primeros estudios sobre el tema $[21,10,14]$ definen el chatter como un modo autoexcitado de vibración, y estudian las posibles causas a través de modelos. En [23], se recogen varios modelos para la estructura del tren y el proceso de laminación, los cuales se combinan para obtener modelos de chatter.

Los modelos estructurales clásicos están basados en un sistema masa-muelle, donde las fuerzas se representan en términos de rigidez y amortiguamiento. Dado que algunos de estos modelos asumen simetría respecto al hueco de los rodillos, so- lo la parte superior se considera para el análisis. Los modelos del proceso de laminación son expresiones matemáticas relacionadas con los parámetros de laminación que ayudan a determinar, por ejemplo, la fuerza de laminación, el par, el punto neutro, la tensión, etc. Todas implican coeficientes tales como el límite elástico y la fricción.

Matemáticamente, puede representarse un modelo simplificado mediante una ecuación diferencial que contiene las dependencias entre variables expresadas en la ecuación 1 (omitiendo referencia explícita a las derivadas de diversos órdenes que puedan aparecer), donde el espesor de salida $\left(h_{s}\right)$ depende de la fuerza de laminación $(F)$, las tensiones de entrada y salida $\left(\sigma_{e}\right.$ y $\left.\sigma_{s}\right)$, las velocidades $\left(\begin{array}{lll}v_{e} & \mathrm{y} & v_{s}\end{array}\right)$ y el espesor de entrada $\left(h_{e}\right)$. El principal objetivo es el estudio de la función $f$ y las variables que afectan internamente al sistema:

$$
h_{s}=f\left(F, \sigma_{e}, \sigma_{s}, v_{e}, v_{s}, h_{e}\right)
$$

Hay más elementos en el proceso que intervienen en el funcionamiento del sistema completo. En [20] se propone un modelo de caja única en espacio de estados, acoplando modelos del proceso dinámico de laminación, la estructura de la caja y el sistema servo hidráulico. Este sistema se simplifica para llevar a cabo un control robusto del espesor. También, el estudio de la estabilidad con respecto a los parámetros de laminación en [4] o el uso de modelos de múltiples cajas en trenes de laminación tipo tándem, como se explica en [24] o en [5], incrementan la complejidad del estudio. Por tanto, aún existen dificultades para una comprensión completa de las condiciones que conducen a la inestabilidad del sistema.

Los algoritmos de análisis de datos pueden utilizarse para estudiar las condiciones dinámicas. Por ejemplo, los mapas topológicos autoorganizados se han utilizado para explorar el comportamiento dinámico de procesos industriales, incluido el análisis de trenes de laminación [3]. También se ha descrito el uso de técnicas de manifold learning para generar mapas visuales de estados de vibración en máquinas rotativas [2] o comportamientos dinámicos de trenes de laminación en frío, proyectados en una visualización estática [11], que puede utilizarse como un mapa de estados de vibración del proceso. En [7] y en [17] se pueden encontrar referencias acerca de cómo los beneficios cognitivos de la visualización pueden ayudar a interpretar de forma eficiente la información.

Aunque estos trabajos aportan mapas para analizar comportamientos dinámicos, carecen de mecanismos de interacción que podrían mejorar drásticamente la exploración de datos. La interacción es 


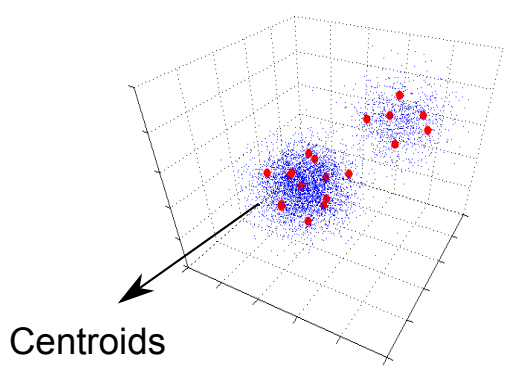

Input space

(dynamic parameters)

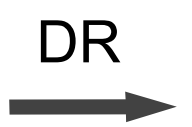

$(\mathrm{t}-\mathrm{SNE})$
Details on demand

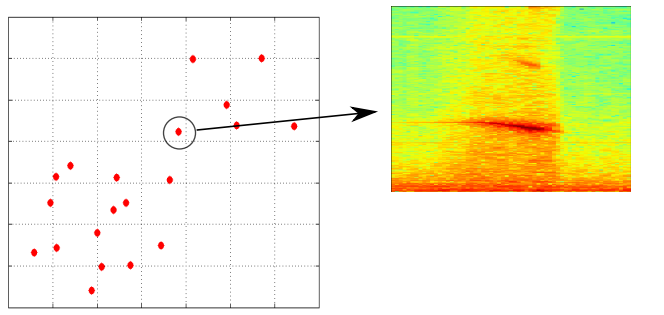

\section{Visualization space}

(user-interaction)

Figura 1: Esquema donde características dinámicas de los datos se proyectan en un espacio de visualización.

una parte clave en los interfaces visuales, porque permite la manipulación y el enfoque selectivo en la representación. Trabajos previos, como [13] o [22], describen taxonomías de los mecanismos de interacción. La combinación entre los algoritmos y los beneficios de la visualización sugieren soluciones basadas en el paradigma de analítica visual para abordar problemas complejos [6].

\section{DESCRIPCIÓN DEL ANÁLISIS}

En esta sección se describe un procedimiento para el análisis de datos del chatter que permite aportar intuición al problema. Aquí se expone un método automático de identificación de condiciones de funcionamiento, una extracción de características y un método para obtener la proyección. En la Fig. 1 se muestra un esquema del enfoque propuesto.

\subsection{IDENTIFICACIÓN DE CONDICIONES DE FUNCIONAMIENTO}

En primer lugar, se llevó una extracción de estados de funcionamiento para identificar condiciones normales y de chatter mediante algoritmos automáticos. Este proceso se lleva a cabo en dos fases:

\subsubsection{Condiciones de chatter}

El algoritmo de identificación de episodios de chatter se basa en el seguimiento del armónico de máxima amplitud de una señal $A(t)$, que puede ser la desviación de espesor de salida o la vibración de una caja, dentro de la banda de chatter usando "Short-Time Fourier Transform" (STFT) o espectrograma. Se considera la posibilidad de existencia de chatter cuando dicho armónico su- pera cierto umbral $A_{c}$. Para confirmar la presencia de un episodio de chatter el algoritmo también se apoya en la identificación de bajadas de velocidad de laminación $v(t)$ presumiblemente realizadas por el sistema de detección de chatter existente en el tren. El funcionamiento del algoritmo depende del ajuste de un cierto número de parámetros, como por ejemplo los límites de la banda de chatter, el umbral para el armónico de máxima amplitud, y otros, hasta completar un número de alrededor de 16. La mayoría de esos parámetros se han ajustado de manera heurística. Una de las razones de hacerlo así es la falta de conocimiento exacto de los detalles del funcionamiento del propio sistema de detección de chatter instalado en el tren. Por tanto es de interés disponer de una herramienta que permita evaluar los resultados del algoritmo y ayudar a la selección de sus parámetros, como se verá más adelante.

En la Fig. 2 se muestra un esquema de este sistema de identificación automático. En el caso de detectarse una condición de chatter, se calcula un coeficiente $\alpha$ ajustando la evolución temporal del armónico analizado a la ecuación $A(t)=K e^{\alpha t}$. Este parámetro puede utilizarse como una medida de la severidad de la inestabilidad producida por el fallo.

\subsubsection{Condiciones normales}

A partir de las bobinas que no contenían episodios de chatter, se implementó una identificación de condiciones normales detectando tramos constantes en la velocidad de laminación de la última caja, descartando todas las variaciones que pueden suceder tales como el aumento y descenso que suceden en inicio y el final del proceso de laminación respectivamente. 


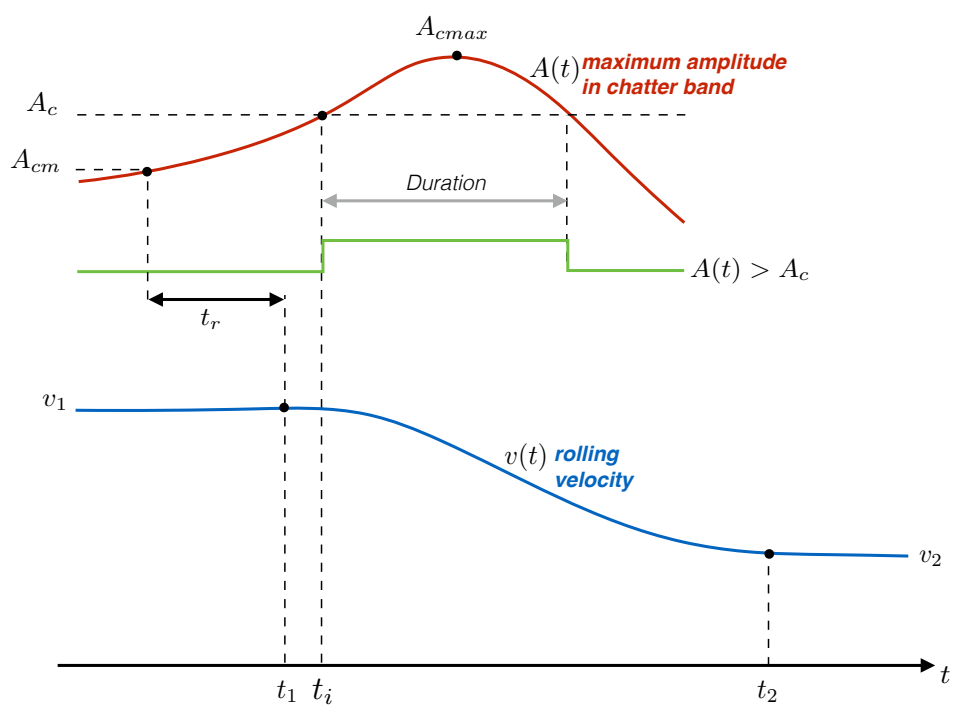

Figura 2: Esquema del método de identificación de chatter.

\subsubsection{Extracción de características}

Finalmente, una vez que ambos tipos de funcionamiento han sido detectados, se tomaron segmentos de 1 segundo para calcular ciertas características en cada condición, para varias variables.

\subsection{MÉTODO DE PROYECCIÓN DE DATOS}

Se implementó un sencillo método para obtener un conjunto manejable para calcular su proyección. En la Fig. 3 se muestra un diagrama de bloques del método implementado.

Dado que existen una gran cantidad de condiciones normales que pueden identificarse, se llevó a cabo un submuestreo, seguido de una etapa de cuantificación vectorial usando el método $k$ means, con el objeto de reducir el conjunto de datos para este tipo de condición. Los centroides resultantes son prototipos representativos de este tipo de funcionamiento del proceso, pero no describen puntos reales del proceso. Con el objeto de trabajar con situaciones reales, se consideraron, por tanto, los puntos más cercanos a los prototipos.

Reuniendo todos los vectores de condiciones normales y de chatter, se construyó una matriz de datos y se normalizó eliminando la media y escalándola de forma que tenga varianza unidad para cada variable.

Se obtuvo una proyección para visualizar las similitudes usando el algoritmo $t$-SNE [18]. Esta técnica es muy superior a las anteriores basadas en esquemas básicos de preservación de distancias. El $t$-SNE, por el contrario, considera la vecindad de los puntos de entrada $\left\{\mathbf{x}_{i}\right\}_{i=1, \ldots, N}$ siendo $\mathbf{x}_{i} \in \mathbb{R}^{D}$, por medio de una matriz de probabilidad $P$, donde:

$$
p_{j \mid i}=\frac{\exp \left(-\left\|\mathbf{x}_{i}-\mathbf{x}_{j}\right\|^{2} / 2 \sigma_{i}^{2}\right)}{\sum_{k \neq i} \exp \left(-\left\|\mathbf{x}_{i}-\mathbf{x}_{k}\right\|^{2} / 2 \sigma_{i}^{2}\right)}
$$

El algoritmo utiliza una transformación simétrica de dicha matriz. En paralelo, las probabilidades de los puntos de baja dimensión, $\left\{\mathbf{y}_{i}\right\}_{i=1, \ldots, N} \in \mathbb{R}^{d}$ con $d<D$ son modelados mediante una distribución t-Student de un grado de libertad, definiéndose $Q$ como:

$$
q_{i j}=\frac{\left(1+\left\|\mathbf{y}_{i}-\mathbf{y}_{j}\right\|^{2}\right)^{-1}}{\sum_{k \neq l}\left(1+\left\|\mathbf{y}_{k}-\mathbf{y}_{l}\right\|^{2}\right)^{-1}}
$$

Las diferencias entre las distribuciones de probabilidad $P$ y $Q$ son evaluadas utilizando la divergencia de Kullback-Leibler:

$$
D_{K L}(P \| Q)=\sum_{j} p_{i j} \log \frac{p_{i j}}{q_{i j}}
$$

El cálculo de los puntos $\mathbf{y}_{i}$ que minimizan esta divergencia permite obtener la proyección resultante.

\section{CASO DE ESTUDIO}

\subsection{Análisis de datos reales}

Para validar el análisis se utilizan datos reales de un tren tándem procedentes de un proceso de laminación en frío. Los algoritmos descritos en la sección 3 se desarrollaron en lenguaje Python utilizando paquetes como pandas, scipy o scikit-learn. 


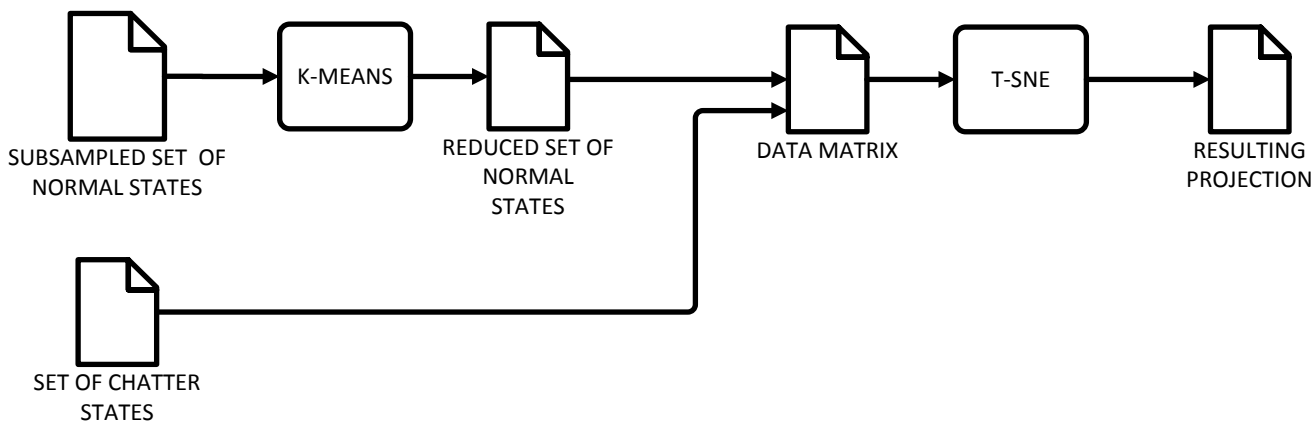

Figura 3: Flujograma para la obtención de la proyección.

Los datos recogidos de un tren tándem de laminación en frío corresponden a una producción de 4102 bobinas. Las variables seleccionadas para caracterizar las condiciones dinámicas incluyen magnitudes como fuerzas, tensiones, velocidades de laminación o reducciones estimadas $\left(r_{i}=1-\frac{v_{i e}}{v_{i s}}\right)$, siendo $v_{i e} \mathrm{y} v_{i s}$ las velocidades de entrada y salida respectivamente en la caja $i$. Estas variables se describen de manera detallada en la Tabla 1. Para obtener la proyección, se consideran todas las variables de la tabla, excepto el número de identificación (1) y el tipo de material (2). Las características utilizadas son los valores medios de segmentos de la señal (de un segundo de duración), excepto en el caso de las vibraciones, para las que se calcularon los valores eficaces (RMS).

Tabla 1: Descripción de las variables.

\begin{tabular}{|c|l|l|}
\hline$\#$ & Descripción & Unidades \\
\hline 1 & Número de identificación & - \\
2 & Tipo de material & - \\
3 & Espesor objetivo de entrada & $\mu \mathrm{m}$ \\
4 & Espesor objetivo de salida & $\mu \mathrm{m}$ \\
5 & Ancho de la bobina & $\mathrm{mm}$ \\
6 & Suma de fuerzas en caja 4 & $\mathrm{t}$ \\
7 & Suma de fuerzas en caja 5 & $\mathrm{t}$ \\
8 & Tensión entre cajas 3-4 & $\mathrm{t}$ \\
9 & Tensión entre cajas 4-5 & $\mathrm{t}$ \\
10 & Velocidad laminación caja 3 & $\mathrm{~m} / \mathrm{min}$ \\
11 & Velocidad laminación caja 4 & $\mathrm{m} / \mathrm{min}$ \\
12 & Velocidad laminación caja 5 & $\mathrm{~m} / \mathrm{min}$ \\
13 & Vibración caja 4 (RMS) & $\mathrm{g}$ \\
14 & Vibración caja 5 (RMS) & $\mathrm{g}$ \\
15 & Desviación espesor de salida & $\%$ \\
16 & Reducción caja 4 & - \\
17 & Reducción caja 5 & - \\
\hline
\end{tabular}

A pesar de no utilizar en este caso características dinámicas realmente, las características escogidas pretenden discriminar entre diferentes puntos de funcionamiento que, al tratarse de un sistema no lineal y variante, corresponderán a diferentes comportamientos dinámicos.

Se aplicó el método explicado en la Sección 3.2 a un conjunto de entrenamiento de condiciones normales con un tamaño de más de 1 millón de observaciones. La operación de submuestreo redujo el conjunto a 10000 muestras y con el algoritmo $k$-means resultan finalmente 1000 centroides, con los que se calculan sus puntos más cercanos. Por otra parte, las condiciones de chatter identificadas fueron 519 episodios, por lo que la matriz de datos resultante tiene unas dimensiones de $1519 \times 15$. Para realizar la reducción de la dimensionalidad, se utilizó el algoritmo $t$-SNE de la librería scikitlearn, con una inicialización PCA, una velocidad de aprendizaje (learning rate) de 900 y una perplejidad (perplexity) de 30, ambas determinadas experimentalmente.

Para calcular los espectrogramas se utilizó la señal de la desviación de espesor de salida, con un segmento de tamaño 1024 y ventana Tukey. Para facilitar su comparación, todos los espectrogramas se calcularon en la misma banda de frecuencias, comprendida entre 0 y $400 \mathrm{~Hz}$ y con la misma escala de color para las amplitudes. Asimismo, se calculó la mayor duración de un episodio de chatter (que resultó ser de 10 segundos) para establecer un rango de tiempos común para todos los espectrogramas.

\subsection{Aplicación web}

La aplicación debería permitir al usuario identificar estados dinámicos similares rápidamente en una vista general donde se puedan identificar las condiciones de fallo fácilmente. Esto se obtiene por medio de la proyección t-SNE de los vectores dinámicos, la cual proporciona un mapa de los estados dinámicos donde puntos cercanos representan condiciones dinámicas similares. La evaluación de estos episodios se puede realizar fácilmente 


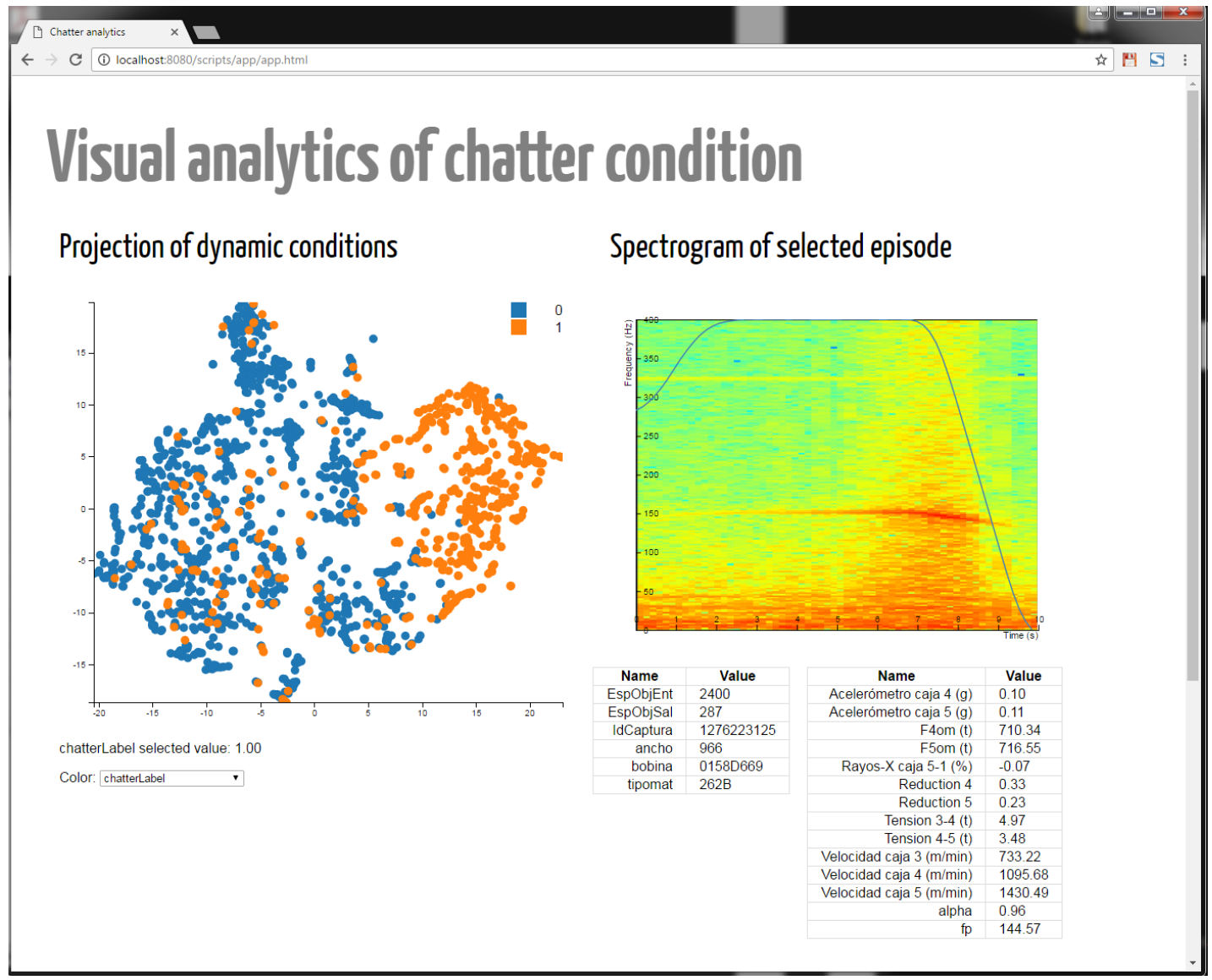

Figura 4: Captura de pantalla de la aplicación mostrando la proyección de los estados dinámicos (izda.), el espectrograma (dcha.) y las tablas.
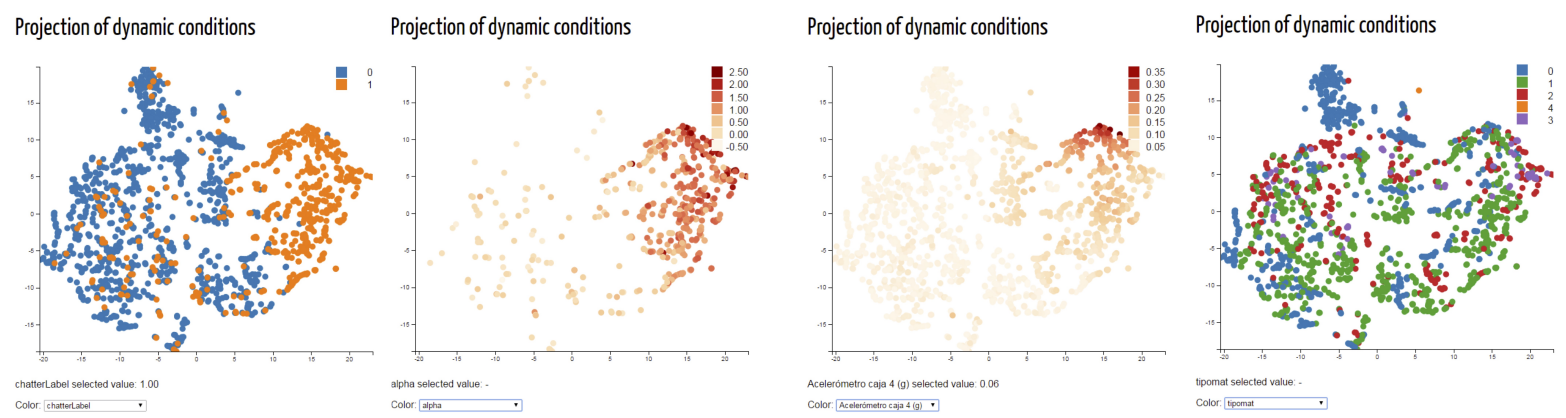

Figura 5: Proyección usando varias escalas de color (de izda. a dcha.): estado normal/chatter, parámetro $\alpha$, vibración en la $4^{a}$ caja y tipo de material. 
a través de los espectrogramas, que aportan una representación tiempo-frecuencia detallada de cada episodio. Esto se puede hacer bajo demanda, estableciendo conexiones entre ambas vistas.

En la Fig. 4 se muestra una captura de pantalla del prototipo web diseñado. La representación de puntos muestra la proyección t-SNE de los estados dinámicos donde el color de los puntos muestra el valor de una determinada variable, con una escala de color predefinida. El usuario puede cambiar esa variable por medio de un selector. Mecanismos de interacción adicionales, como zoom o pan, están disponibles para facilitar la exploración en la proyección.

Gracias al principio de espacialización proporcionado por el algoritmo t-SNE (cercano similar), los estados de chatter parecen estar más aislados que el resto de las condiciones normales de operación, haciéndolos visualmente identificables por el usuario. Sin embargo, algunas excepciones de episodios que fueron etiquetados como chatter en etapas previas, se situaron en el área del mapa de condición normal, donde evaluaciones más detalladas pueden llevarse a cabo utilizando esta aplicación.

Moviendo el puntero del ratón sobre los puntos de la proyección, se resalta el punto seleccionado y el valor exacto de la variable del color se muestra en un texto debajo. Además, si es un episodio de chatter, el espectrograma calculado se muestra a la derecha. La línea azul sobre el espectrograma indica la velocidad de laminación de la última caja. Todos los espectrogramas, se calcularon con las mismas especificaciones, entre 0 y $400 \mathrm{~Hz}$ y un rango de tiempo de 10 segundos, como se indicó previamente. Los mismos mecanismos de zoom y pan se pueden aplicar al espectrograma, permitiendo al usuario navegar en los detalles de la imagen. Esto ayuda al usuario a confirmar, de manera visual, la existencia de un episodio de chatter identificado de manera automática previamente.

Debajo de la imagen del espectrograma hay dos tablas que muestran los detalles del punto elegido. La tabla de la izquierda incluye parámetros característicos de la bobina, como el ancho, tipo de material, o espesor objetivo. La tabla de la derecha muestra valores de la condición dinámica como los valores medios.

Mientras el usuario navega por los puntos proyectados, el espectrograma y los valores de las tablas se actualizan de manera instantánea. Esto permite una rápida exploración de las condiciones dinámicas y la evaluación de los detalles de cada episodio de chatter, previamente identificado, que el usuario seleccione.

En la Fig. 5 se muestra la proyección con diferentes escalas de color representando varias variables (de izquierda a derecha): el estado de normal/chatter, parámetro $\alpha$, vibración en caja 4 y tipo de material. La comparación entre las distintas representaciones permite encontrar relaciones entre variables. Por ejemplo, puede apreciarse cómo la figura muestra al usuario visualmente que el parámetro $\alpha$ tiene valores más altos para los estados de chatter dentro del área de chatter o que la mayor vibración de la caja 4 corresponde a parte de los estados de chatter.

\section{CONCLUSIONES}

En este artículo se propone un enfoque basado en el paradigma de analítica visual para un análisis exploratorio de condiciones de chatter. Se procesaron datos reales procedentes de un proceso de laminación en frío para identificar condiciones de funcionamiento normales y de chatter de forma automática. Se calcularon vectores compuestos por 15 descriptores del proceso para caracterizar ambos tipos de estados dinámicos. A partir de estos vectores se realizó una proyección $2 \mathrm{D}$ utilizando el algoritmo $t$-SNE, que aporta una visualización eficaz de los estados y a la vez permite mostrar de forma rápida los espectrogramas correspondientes a los estados de chatter, mediante eventos tipo hover.

Se desarrolló una aplicación web para exploración interactiva de datos que ayuda al usuario a obtener una vista general de las condiciones normales y de chatter en el proceso, que al mismo tiempo aporta detalles bajo demanda de cualquier episodio de chatter identificado. De esta manera, se han revisado visualmente episodios previamente detectados como chatter de manera automática que resultan dudosos, pudiéndose evaluar el grado de severidad del episodio. Por tanto, esto puede ser utilizado como herramienta para una validación visual del fallo del chatter, así como método de apoyo para el refinado experimental de los parámetros involucrados en el algoritmo de detección automática de chatter.

Las líneas de trabajo futuro incluyen el desarrollo de vistas coordinadas, dotadas de nuevos mecanismos de interacción como el filtrado, que permitan al usuario seleccionar las partes interesantes de los datos para descubrir conocimiento nuevo acerca de los fallos.

\section{Agradecimientos}

Este trabajo ha sido financiado por el Ministerio de Economía y Competitividad (MINECO), Programa Estatal de I+D+i Orientada a los Retos de la Sociedad y por el Fondo Europeo de Desarrollo Regional (FEDER) "Una manera de hacer 
Europa", bajo el proyecto de referencia DPI201569891-C2-2-R.

\section{Referencias}

[1] J. Alexander. On the theory of rolling. In Proceedings of the Royal Society of London A: Mathematical, Physical and Engineering Sciences, volume 326, pages 535-563. The Royal Society, 1972.

[2] I. Díaz, A. A. Cuadrado, A. B. Diez, and M. Domínguez. Manifold learning for visualization of vibrational states of a rotating machine. In ICANN (2), pages 285-292, 2011.

[3] I. Díaz, M. Domínguez, A. A. Cuadrado, and J. J. Fuertes. A new approach to exploratory analysis of system dynamics using som. applications to industrial processes. Expert Systems with Applications, 34(4):2953-2965, 2008.

[4] A. Heidari and M. R. Forouzan. Optimization of cold rolling process parameters in order to increasing rolling speed limited by chatter vibrations. Journal of Advanced Research, 4(1):27-34, Jan. 2013.

[5] P.-H. Hu and K. F. Ehmann. Stability analysis of chatter on a tandem rolling mill. Journal of Manufacturing Processes, 2(4):217$224,2000$.

[6] D. A. Keim, F. Mansmann, J. Schneidewind, J. Thomas, and H. Ziegler. Visual analytics: Scope and challenges. Springer, 2008.

[7] J. H. Larkin and H. A. Simon. Why a diagram is (sometimes) worth ten thousand words. Cognitive science, 11(1):65-100, 1987.

[8] J. A. Lee and M. Verleysen. Nonlinear dimensionality reduction. Springer, New York; London, 2007.

[9] E. Orowan. The calculation of roll pressure in hot and cold flat rolling. Proceedings of the Institution of Mechanical Engineers, 150(1):140-167, 1943.

[10] D. L. Paton and S. Critchley. Tandem mill vibration: Its cause and control. In Mechanical Working; Steel Processing XXII, Proceedings of the 26th Mechanical Working; Steel Processing Conference., pages 247-255, Chicago, IL, USA, 1985. Iron and Steel Soc Inc.

[11] D. Pérez, F. García-Fernández, I. Díaz, A. Cuadrado, D. Ordonez, A. Díez, and M. Domínguez. Visual analysis of a cold rolling process using a dimensionality reduction approach. Engineering Applications of Artificial Intelligence, 26(8):1865-1871, 2013.

[12] W. L. Roberts. Cold rolling of steel. Marcel Dekker, Inc., New York, 1978.

[13] B. Shneiderman. The eyes have it: A task by data type taxonomy for information visualizations. In Visual Languages, 1996. Proceedings., IEEE Symposium on, pages 336-343. IEEE, 1996.

[14] T. Tamiya, K. Furui, and H. Iida. Analysis of chattering phenomenon in cold rolling. In International Conference on Steel Rolling,, volume 2, pages 1191-1202, 1980.

[15] J. J. Thomas and K. A. Cook. Illuminating the path: The research and development agenda for visual analytics. IEEE Computer Society Press, 2005.

[16] J. Tlusty, G. Chandra, S. Critchley, and D. Paton. Chatter in cold rolling. CIRP Annals-Manufacturing Technology, 31(1):195-199, 1982.

[17] E. R. Tufte and P. Graves-Morris. The visual display of quantitative information, volume 2 . Graphics press Cheshire, CT, 1983.

[18] L. Van der Maaten and G. Hinton. Visualizing data using t-SNE. Journal of Machine Learning Research, 9:2579-2605, 2008.

[19] C. Ware. Information visualization: perception for design. Elsevier, 2012.

[20] X. Yang and C.-n. Tong. Coupling dynamic model and control of chatter in cold rolling. Journal of Dynamic Systems, Measurement, and Control, 134(4):041001, 2012.

[21] I. Yarita. Analysis of chattering in cold rolling for ultra-thin gauge steel strip. Trans. Iron Steel Inst. Jpn., 18(1):1-10, 1978.

[22] J. S. Yi, Y. ah Kang, J. T. Stasko, and J. A. Jacko. Toward a deeper understanding of the role of interaction in information visualization. Visualization and Computer Graphics, IEEE Transactions on, 13(6):12241231, 2007.

[23] I. S. Yun, W. R. D. Wilson, and K. F. Ehmann. Review of chatter studies in cold rolling. International Journal of Machine Tools and Manufacture, 38(12):1499-1530, 1998.

[24] H. Zhao and K. F. Ehmann. Stability analysis of chatter in tandem rolling mills?part 1: single-and multi-stand negative damping effect. Journal of Manufacturing Science and Engineering, 135(3):031001, 2013. 